\title{
DESIGN OF MOBILE SHORT MESSAGE SERVICE (SMS) ACROSS A COMPUTER NETWORK FOR ORGANISATIONAL COMMUNICATION
}

Jimoh, R. G.

Dept. of Computer Science

University of Ilorin, Nigeria
COCO, K. B

Dept. of Computer Science

University of Ilorin, Nigeria

\author{
OLUOBO, Abdel, M. F. O. \\ Department of Computer Science \\ University of Ilorin
}

\author{
Abdulsalam, S. O \\ Dept. of Computer, Library \& Info. Science \\ Kwara State University, Malete, Nigeria
}

Isiaka, R. M.

Dept. of Computer, Library \& Info. Science

Kwara State University, Malete, Nigeria

\begin{abstract}
Short Message Service (SMS) is the most powerful tool in terms of communication especially for mobile users. It does not limit anyone regardless of high- or low-end mobile phones for as long as they can receive and send messages anytime, anywhere. It was revealed that, lack of adequate communication technology in an organization leads to a number of issues that make such organization to perform less. In order to fully utilize the mobile phones, this study has come up with a fast way where users can get information quickly without spending more. This study aimed to promote access to such information through the use of Short Message Service (SMS) and improve transparency, reliability, usability of the information for organization. The proposed SMS application will provide multi level local authentication to the SMS gateway service. The application was developed based on the Unified Software Development Process or in short, the Unified Process being a components-based system. The programming languages used are PHP and JAVA. The resulting SMS application is found to be usable and economical.
\end{abstract}

Keywords: SMS, SMS gateway, Transparency, Reliability, Usability.

\section{INTRODUCTION}

The Short Message Service (SMS) allows textual messages to be delivered between SMS enabled and digital cell phones from a computer network. These messages travel on the network in tandem with voice call signaling traffic. The service is extremely popular in Europe and Asia, generating tens of billions of messages a month. A number of factors hindered initial SMS acceptance in the United States, but traffic has grown remarkably in the last two years, and this trend is expected to continue [3]. SMS has also demonstrated to be a reliable alternative to voice communications, during the terrorist attacks on September 11, 2001. High traffic volumes made it extremely difficult to connect people on calls; however, SMS text messaging continued to operate and provide communication means for people who understand how to use it [3].

The conflux of these events raises the question: given that SMS shares the network, that popularity and awareness of SMS are increasing rapidly, and that SMS might be a person's means of communications during another crisis, how would the wireless network handle the surge in short messaging traffic? [7]. This question was answered by wireless network reliability, capacity and congestion handling, security and vulnerability, priority services, and new SMS-related developments. It was then concluded that networks are theoretically capable of continued operation and service (including NS/EP service) during a crisis [7]. However, it may be possible for extremely high volumes of SMS traffic, when combined with high numbers of voice call attempts, to interfere with a wireless network's performance. The absence of a definitive conclusion is due to the unavailability of key information: the network configuration details (known only by the network operator), the congestion handling algorithms (known only by the equipment manufacturers). Organizations today are facing several unique communication challenges: the proliferation of electronic messages across their enterprise; the need to provide employees, customers, and partners with consistent information; the desire to better equip information workers with meaningful information to drive informed decisions; and the mandate to control costs without sacrificing application availability, security, or reliability [5]. Popularity, will impact the future load on SS7 [3]. This Technical Information Bulletin (TIB) addresses two major SMS Markets; the United States and Europe. In Great Britain alone, over 68 million short messages were sent on Valentine's Day, 2003. In Europe, 10 billion messages are sent each month. In the United States, SMS traffic has increased tremendously to today where hundreds of millions of short messages are sent each month. The goal TIB is to:

1. Perform an analysis of the vulnerability of SS7 to a huge increase in Short Messaging Service (SMS) traffic. 
2. Examine the state-of-the-art of SMS applications in the PSN environment.

3. Examine the security aspects of employing SMS in an NS/EP environment.

4. Provide an analysis of future developments in SMS and transmission media other than PSN.

TIB includes:

- A description of the SMS technology.

- Capacity and load analysis for high traffic volume situations.

- $\quad$ Security issues of using SMS in the PSN and other media.

All these features among others are captured in this work.

\section{PROBLEM STATEMENT}

It is generally believed that information is power. At the same time, useful information cannot be gathered without effective communication. Efforts on effective communication strategy should therefore be continuous. SMS has become an alternative to voice communication as it has been revealed by previous authors that, a number of factors can hinder effectiveness of the voice communication method [7]. It was revealed that, lack of adequate communication technology in an organization leads to a number of issues that make such organization to perform less [5]. The researcher is interested in filling this gap by proposing a usable mobile SMS for organizational communication.

\section{RESEARCH QUESTIONS AND OBJECTIVES}

1. What are the previous SMS-based communication strategies?

2. How can a mobile SMS-based communication prototype be developed?

The aim of this research work is to produce a useful program that takes care of mobile short message service (SMS) for organizational communication. In achieving this central objective, the following specific objectives are formulated:

1. To review existing SMS communication strategies

2. To design and develop SMS approach as a means of communication in an organization

\section{SOFTWARE DEVELOPMENT APPROACH USED}

The software development approach used in this research work is the Unified Software Development Process or simply put, the Unified Process (USDP). USDP was used because it is a component-based process which allows various components making up the system to be linked together through a well defined interface. Information systems are embedded in an organizational context. As the organization surrounding the system evolves, new requirements emerge. The implementation of a new system can also transform the usage context and therefore new requirements arise [2].The evolutionary prototyping approach results in the gradual development of systems and allows for, or adapts to, changes that take place within an organization as a result of either the operationalisation of the system, or externally induced changes to the organization [4].In contexts of high uncertainty (and unlike contexts with high certainty where waterfall models are employed), the evolutionary prototyping approach dynamically responds to changes in user needs and accommodates subsequent unpredictable changes in requirements, as the development process progresses [6].

[2] identifies incremental development and evolutionary system development as two distinct approaches that employ prototypes. During incremental development the system evolves gradually in partial increments against the backdrop of an overall long range development strategy. The fundamental difference between evolutionary development and incremental development is in system design; while software design evolves in evolutionary development, there are no changes to the design during incremental development [4]. Whilst incremental development assumes that most user needs are known at the beginning, in evolutionary system development, the prototype is built in an area where overall the requirements are not well understood [1]. Initial development, however, starts in an area where the requirements are known. USDP allows all this components making up a system to be well represented using a Unified Modeling Language. In fact, The Unified Process uses Unified Modeling Language (UML) in preparation of blueprints for the software.

Unified Process is more than a single process; it is a generic process framework that can be used for a very large class of software systems, for different application areas, different types of organizations, different competence levels and different project sizes. The Unified Process is component-based which implies that the software system being built is made up of software components interconnected via well-defined interfaces as described in Figure 1.

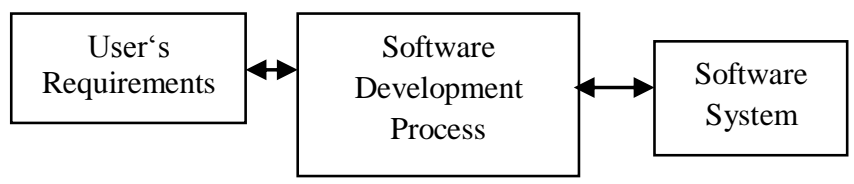

Figure 1: Software Development Process. 


\section{SYSTEM ARCHITECTURE}

Figure 2 describes the system architecture of the proposed SMS system. The components developed by team members are in the rectangular with dash line.



Figure 2: SMS Spot Application Architecture

The application contains seven main components. Its functions are described as follows:

1. Web Browser: Running on client-side, it allows users $\log$ in and send SMS messages.

2. Web Service: Provide the service for gateway and SMS application to send and receive SMS messages under JAXWS

3. EVI (Enterprise Voice Integration) profile: contain the information of customers. Based on this information, the web application can determine the route and protocol for transferring a message.

4. Web Application: Running in the server-side, it handles requests from SMS users for sending and receiving SMS message. Beside, the web application can get the information from EVI profile or database, and then it decides the route and the protocol that it uses to send the SMS message. This is the server side component to be built by the project.

5. SMS Gateway: an SMS gateway can be set up to handle the connections to the SMSCs. To connect to an SMS gateway, an SMSC protocol is used such as SMPP, HTTP/HTTPS, email to SMS, etc.

6. SMS Center: A SMS center (SMSC) is responsible for handling the SMS operations of a wireless network. When an SMS message is sent from a mobile phone, it will reach an SMS center first. The SMS center then forwards the SMS message towards the destination.

7. Mail Server: Provide SMTP service to send SMS messages to the mail system of the mobile phone carriers and a mailbox to receive the SMS message sent from mobile phones. 
International Journal of Computer Applications Technology and Research

Volume 2- Issue 4, 409 - 414, 2013, ISSN: 2319-8656

\section{USE CASE DIAGRAM OF THE PROPOSED SYSTEM}

Use Case diagram of the application is shown in Figure 3. It contains three actors which are User, Administrator and Mobile and the functions performed by these actors
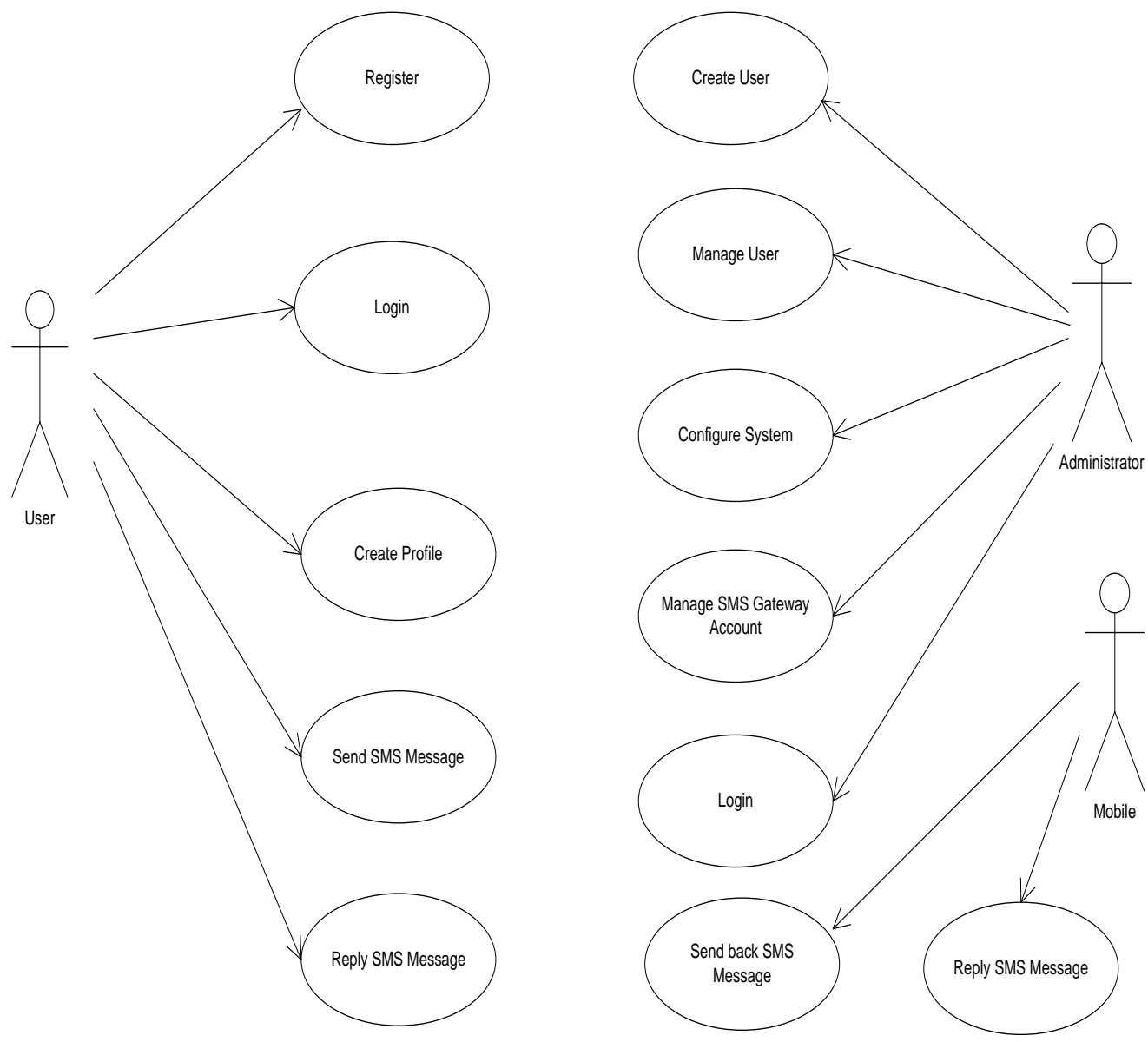

Figure 3 SMS Live Spot Use Case Diagram

\section{CLASS DIAGRAM OF THE PROPOSED SYSTEM}

The Database includes four tables which are Message, Contact, Group and GroupDetail to provide complete information to users and they are stored in MySQL database management system as shown in Figure 4. Message table: is identified by MessageID and contains information such as messagetype, subject, content, sender, date and userID. This table is used to store all the messages sent back and forth by users. Contact table is identified by UserID and contains information such as Username, password, firstname, lastname, address, homephone, cellphone and servprovider. This table is used to store the contact or recipient information that user want to send messages to. Group table is identified by userID, groupID and contains information such as group name, description. This table is used to store group of contact or recipient information that user want to send messages to. GroupDetail table is identified by userID, groupID. This table is used to provide detail information of the corresponding group. 
International Journal of Computer Applications Technology and Research

Volume 2- Issue 4, 409 - 414, 2013, ISSN: 2319-8656

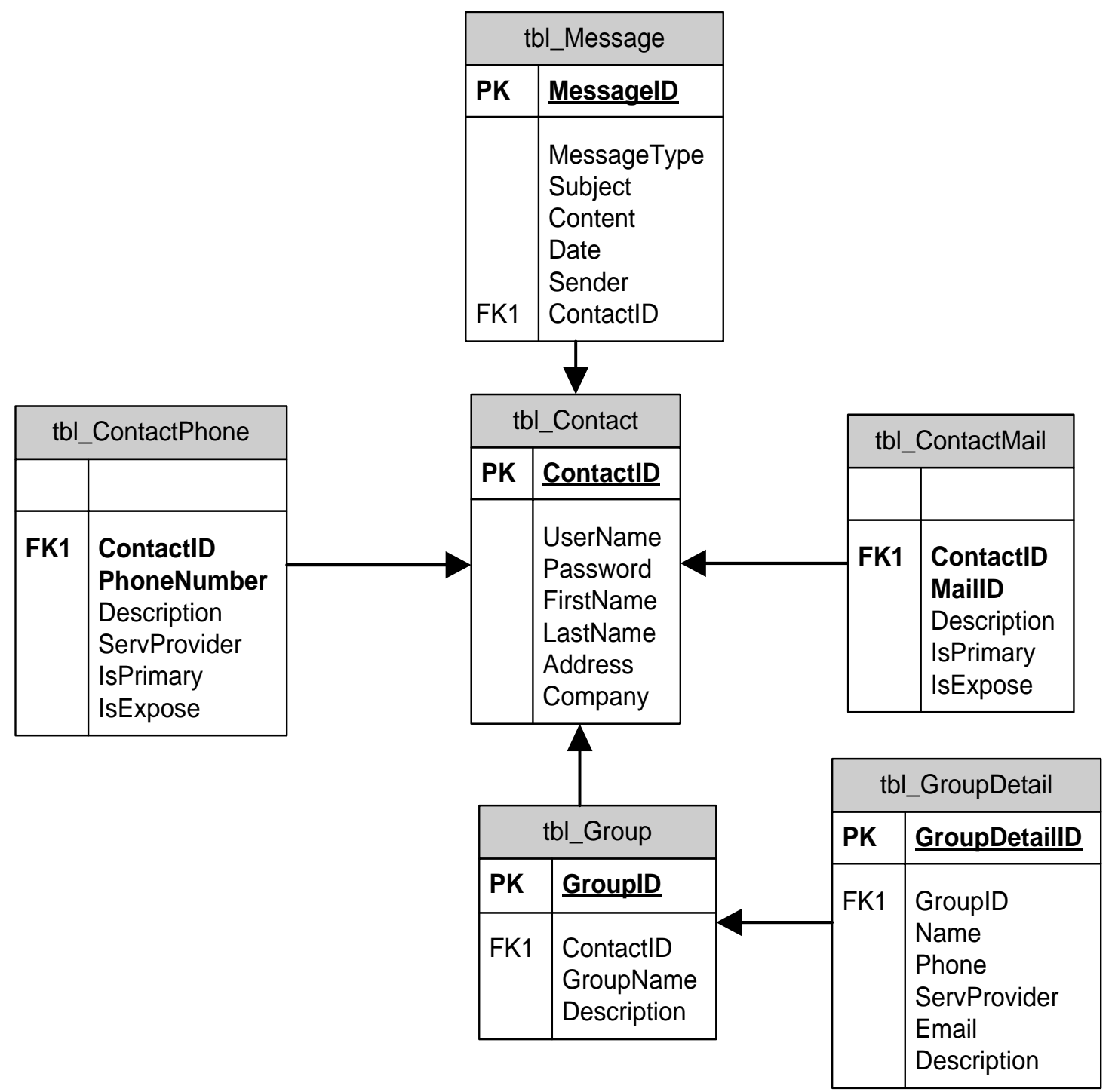

Figure 4: Class Diagram

\section{DISCUSSION}

The following prototype is developed based on the design described earlier and the usability of the developed prototype was also confirmed. Communication is very essential in virtually every organization. The emerging knowledge-driven organization places communication more prominently in achieving the objectives of such organization. Communication takes place at every stage in an organization. Various efforts have been made in making communication experience within an organization an interesting one. This work is contributing in this direction by proposing a mobile SMS across a computer network for a communication platform and also provides such service at almost free rate since it is across the network. The proposed communication medium is devoid of all barriers associated with communication using ICT as long as the mobile phone is on. This will assist the emerging knowledge-driven organizations in achieving their objectives with prompt and easy access to information. 


\section{CONCLUSION}

Communication has become the life wire of any organization, most especially in this information age. Effectiveness of this communication depends on the reliability of the medium used. With due consideration to all design issues of a typical SMS services, the proposed SMS system has been validated to be reliable in terms of its capacity, Signaling Network Reliability, Signaling Path Reliability, Cost, Security and Privacy. It can therefore be concluded that the SMS system is capable of delivering effective communication within the organization. It is one thing to propose a communication medium, it is another

\section{REFERENCES}

[1] Davis, A., Bersoff, E.H. \& Comer, E.R. (1988). A Strategy for Comparing Alternative Software Development Life Cycle Models. IEEE Transactions on Software Engineering, 14, 14531461.

[2] Floyd, C. (1984) .A Systematic Look at Prototyping, in: Budde, R.,Kuhlenkamp, K., Mathiassen, L. \& Zullighoven, H. (Eds.) Approaches to Prototyping, Heidelberg: SpringerVerlag, 1-17.

[3] Government of Canada Office of Critical Infrastructure Protection and Emergency Preparedness. (2002). Incident Analysis: The September 11, 2001 Terrorist Attacks - Critical Infrastructure Protection Lessons Learned. Quebec: Canada Government thing for it to be affordable enough in terms of the cost since most organizations have the business initiative of saving cost. The proposed system is found to be suitable in this regards. From the past literature reviewed, it can be seen that, research efforts on inexpensive means of communication without compromising reliability should be encouraged. It is recommended that future researchers should concentrate on ways of preventing the likely vulnerabilities to the proposed communication approach.

[4] Ince, D.C. \& Hekmatpour, S. (1987) .Software Prototyping: Progress and Prospects. Information and Software Technology, 29(1), 8-14.

[5] Product Guide.(2006). Retrieved March 16, 2006, from http://www.ozeki.hu/attachement/185/Product guide 2006.03.16.pdf /ms6. p185.

[6] Pape, T.C. \& Thoresen, K. (1992) .Evolutionary Prototyping in a Change Perspective. Information Technology and People, 6, 2-4.

[7] SMS over SS7-NCS. Technical Information Bulletin: National Communication System. (2003). Retrieved April 25, 2012. From http://www.ncs.gov/library/tech.../2003/tib_0302.pdf 